\title{
Biomedical Technologies in the Treatment of Skin and Soft Tissue Defects in Patients with Diabetic Foot Syndrome
}

\author{
Magomed D. Dibirov, MD, $\mathrm{PhD}, \mathrm{ScD}^{1}$; Rasul U. Gadzhimuradov, $\mathrm{MD}, \mathrm{PhD}, \mathrm{ScD}^{1}$; \\ Konstantin A. Koreiba, MD, $\mathrm{PhD}^{2,3 *}$; Aidar R. Minabutdinov, $\mathrm{MD}^{2,3}$ \\ ${ }^{1}$ Moscow State University of Medicine and Dentistry, Russia \\ ${ }^{2}$ Kazan State Medical University, Russia \\ ${ }^{«}$ ¿Diabetic Foot» Center, Kazan, Russia
}

\begin{abstract}
The aim of our study was to investigate the effectiveness of collagen implants in the closure of tissue defects. We offer a method that enables us to avoid the drawbacks of autodermoplasty based on the free split-thickness skin graft.

Materials and Methods: This paper describes all steps of treatment of skin and soft tissue defects in patients with diabetic foot syndrome (DFS), including ultrasonic cavitation with hydrosurgical debridement to remove necrotic debris, purulent pellicle and bacterial biofilm, and an alternative technique for wound defect closure using high-tech biomaterials based on type I collagen ("Collost", "Salvecoll").

Results: Use of type I collagen ("Collost", "Salvecoll") as a component of combination treatment of tissue defects in DFS allowed us to increase the relative rate of wound healing, reduce the incidence of high-level amputations, and significantly reduce inpatient surgical coverage around the clock and speed up a patient's transfer to outpatient care.

Conclusion: Ultrasonic cavitation with hydrosurgical debridement is the most effective procedure for wound preparation for closure. The use of bioplastic collagen materials in patients with DFS is the most effective solution in the management of wound defects.(Int J Biomed. 2016; 6(1):41-45.).
\end{abstract}

Key words: diabetic foot syndrome; ultrasonic cavitation with hydrosurgical debridement; wound defect closure; bioplastic collagen materials; autodermoplasty.

\section{Introduction}

In 2014, worldwide, 387 million people had diabetes mellitus (DM), and its prevalence estimates tend to increase. According to statistical data, nearly $25 \%$ of DM patients suffer from DFS $[1,2]$. Based on analysis of our early data, we can confidently conclude that a rise in the number of DM patients is accompanied by a concomitant growth of patients with trophic skin lesions in the lower limbs. The number of patients with wound defects corresponding to wound depth stages W1, W2 and W3 (based on Wagner's classification) [3,4] was 43 in 2010, 117 in 2012, and 218 in 2014 (Figure 1). DFS is a condition with pathological changes of the peripheral nervous system, arterial bed and microvasculature, and osteoarticular

*Corresponding author: Konstantin A Koreiba, MD, PhD, Associate Professor. Department of General Surgery, Kazan State Medical University, Russia; "Diabetic Foot" Center, Kazan, Russia. diabetstopa5gb@mail.ru structures representing a direct threat to the development of ulcerative and necrotic processes and gangrene of the foot [5], and leads to organ-resecting operations in $85 \%$ of cases [6].

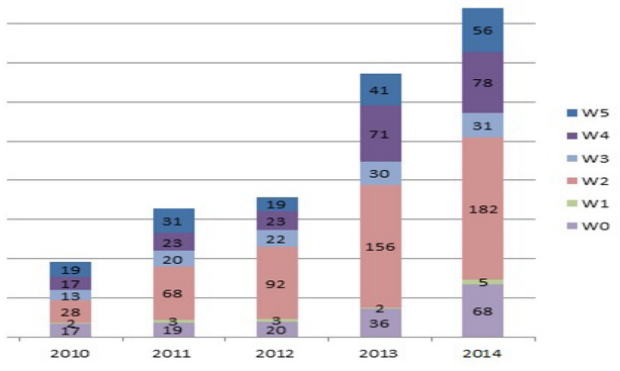

Fig. 1. The number of patients with foot lesions (based on F.W. Wagner's lassification, 1979) for 2010-2014 who received treatment at the "Diabetic foot" center in Kazan

Autosympathectomy (loss of sympathetic nerve innervations), macroangiopathy (initial atherosclerotic lesions 
of peripheral vasculature, Monckeberg's calcific sclerosis and diffuse intimal fibrosis) and microangiopathy (qualitative and quantitative changes of the capillary basement membrane with excessive matrix production and excess permeability) lead to reduced tissue perfusion $[7,8]$. The balance between different protein fractions is disturbed in DFS patients. Of particular importance is the disorganization of collagen, which plays an exceptionally important role during the proliferative and remodeling phases of the wound healing process $[9,10]$.

Collagen is a major component of the ECM and is a prominent target of non-enzymatic glycation with formation advanced glycation end products (AGEs) which play an important role in the pathogenesis of diabetic complications like a DFS [11,12].

Type 1 collagen undergoes a series of post-translational modifications that occur during non-enzymatic glycation. Electron microscopic investigation has revealed fine structural changes in the collagen fibrillar arrangement in diabetes [10]. These differences included increased packing density of collagen fibrils, decreases in fibrillar diameter, and abnormal fibril morphology showing collagen fibrils that appeared twisted, curved, overlapping, and otherwise highly disorganized.

Collagen deposition in acute wounds is impaired in type 1 diabetes, possibly due to a decreased fibroblast proliferation [13]. Overall, collagen enhances the wound contracture and cellular migration that are essential for wound healing. Dynamic interactions between growth factors and extracellular matrix (ECM) are integral to wound healing [14]. Thus, collagen disorganization leads to a disturbed physiological course of wound healing and chronic diabetic foot ulcers.

Upon admission to the Diabetic Foot Center in Kazan, each patient underwent a necessary diagnostic program that included the following:

1. Determination of the severity of diabetic polyneuropathy in accordance with the neuropathy disability score (NDS) developed by M.J. Young in 1986 and recommended by the Diabetes Neuropathy Study Group (NEURODIAB) of the European Association for the Study of Diabetes (EASD).

2. Transcutaneous measurement of tissue oxygen $\left(\mathrm{TcpO}_{2}\right)$.

3. Color-coded duplex ultrasonography (TCCS) of lower limb arteries.

4. X-ray examination of the affected foot.

Based on the examination data, all patients were assigned to two groups. Group 1 included patients with the neuroischemic form of DFS and stage III-IV chronic arterial insufficiency (CAI), who required an immediate arterial reconstruction. Group 2 included patients with the neuroischemic form of DFS and stage I-II CAI and trophic soft tissue lesions, who had no indications for revascularization.

Following the angiographic examination of lower limb arteries, we jointly selected the type of reconstructive surgery procedure according to TASC-II lesion classification and recommended interventions (2007).

The autodermoplastic reconstruction using a free split-thickness skin graft is one of the methods of wound defect closure. However, this treatment procedure in DFS patients is not valid for a number of reasons. The outcome of wound defect closure is not always successful, and is technically difficult to perform on certain areas of the foot. Besides, with the underlying diabetic polyneuropathy, micro- and macroangiopathy, the procedure triggers some pathomorphological mechanisms creating a high risk of an additional chronic wound defect occurring at the skin graft donor site. For that reason, in 2007 a clinical application of high-tech biomaterials based on type I collagen and an investigation of its effectiveness on the wound healing process was started.

\section{Materials and Methods}

The study was conducted in accordance with ethical principles of the Declaration of Helsinki. Written informed consent was obtained from all participants. The clinical data were obtained from 173 FDS patients (mean age 56.4 \pm 15.6 years; 64 men and 109 women). Ulcer defects had different anatomical locations over the lower extremities, namely, on the plantar and dorsal surfaces of the foot, the plantar surface of the fingers, inner or outer surface of the lower third of the tibia and in the area of postoperative wounds of the foot. The area of ulcer defect was, on average, $38.5 \pm 26.3 \mathrm{~cm}^{2}$. According to Wagner Ulcer Grade Classification System, the ulcers were found to be in grade W1, W2 or W3.

The management of wound healing was performed with due consideration of general and local clinical manifestations, such as the general state of patients, intensity of pain, time course of wound bed detersion and granulation tissue formation, start of epithelialization, and changes in the rate of wound contraction. All patients received the same medicinal treatment against the backdrop of hypoglycemic therapy.

On the first day of patient hospitalization, we used ultrasonic cavitation with hydrosurgical debridement to make sure the wound was free of devitalized tissue. This exerts a favorable local effect on the wound healing process and helps to prepare the wound surface for biomaterial implantation. The procedure enabled us to selectively remove necrotic debris, purulent fibrinous pellicle, and devitalized tissue containing bacterial biofilm. The mechanical necrectomy was performed using an equipment for ultrasonic cavitation with hydrosurgical debridement. As a wound-rinsing fluid, we used a weak solution of local anesthetic instead of an antiseptic solution. Our empirical finding was that UC has a major impact on the bacterial biofilm and devitalized tissue whereas the anesthetic solution supplied through the nozzle serves to remove and wash off the ultrasonically cut tissues. The topical anesthesia has undeniable advantage and does not require using the infiltration anesthesia by paravulnar injection of anesthetic or general anesthesia.

After cleansing the wound defect from devitalized tissues, we used high-tech biomaterials based on type I collagen ("Collost", Russia / "Salvecoll", Europe) for optimal coating of skin and soft tissue defects [15]. Collost/ Salvecoll is a type I collagen-based material derived from the skin of cattle and processed in such way that the epidermis, fat tissue and all dermal cells are removed without destroying the collagen matrix. Collost/Salvecoll is devoid of foreign antigens and, 
therefore, of immunogenic properties [16]. For implantation, we used collagen material in the form of membranes $(60 \times 50 \times 1.5 \mathrm{~mm})$, which were applied to the wound bed, and $7 \%$ or $15 \%$ gel, which was injected into the wound bed and walls and to the paravulnar tissues while the needle was being taken out. After the implantation of biomaterial, the wound surface was covered with a hydrocolloid dressing enabling us to maintain a physiological moist environment at the wound/dressing interface. A wound dressing was changed, on average, once in 3-5 days. From day 10-12 post-implantation, we switched to the atraumatic dressing in combination with a coating containing $\mathrm{Ag}^{+}$ions. The frequency of dressing changes depended on the amount of wound exudate and the reaction to the dressing adhesive. Patients were transferred to outpatient care on day 7-10 since the day of biomaterial implantation. Statistical analysis was performed using statistical package for the social sciences (SPSS 15.0 software).

\section{Results}

The epithelialization of wound defect began on $10.3 \pm 2.8$ days. On days 14-20, we could see wound granulation tissue without signs of inflammation or rejection of the bioplastic material. Then, tissue regeneration occurred via formation of the body's own granulation tissues. In none of these cases did we observe hypertrophic scars. Application of Collost / Salvecoll in the treatment of DFS patients allowed us to reduce the average number of bed days by more than $20 \%$ (Table 1 ).

Table 1.

Comparative average number of bed days among DFS patients in "Diabetic Foot" Center and septic surgery departments in Kazan

\begin{tabular}{|c|c|c|c|c|c|}
\hline \multicolumn{6}{|c|}{ "Diabetic Foot" Center } \\
\hline Year & 2010 & 2011 & 2012 & 2013 & 2014 \\
\hline $\begin{array}{l}\text { Average number of } \\
\text { bed days }\end{array}$ & $\begin{array}{c}18.21 \\
\pm 1.2039 \\
\end{array}$ & $\begin{array}{l}15.95 \\
\pm 0.7801 \\
\end{array}$ & $\begin{array}{c}15.29 \\
\pm 1.1434 \\
\end{array}$ & $\begin{array}{c}14.66 \\
\pm 0.4533\end{array}$ & $\begin{array}{c}12.13 \\
\pm 0.3723 \\
\end{array}$ \\
\hline \multicolumn{6}{|c|}{ Septic surgery departments } \\
\hline Year & 2010 & 2011 & 2012 & 2013 & 2014 \\
\hline $\begin{array}{l}\text { Average number of } \\
\text { bed days }\end{array}$ & $\begin{array}{c}21,35 \\
\pm 1.0420\end{array}$ & $\begin{array}{c}20,74 \\
\pm 1.1467\end{array}$ & $\begin{array}{c}20,21 \\
\pm 1.2039\end{array}$ & $\begin{array}{c}19,52 \\
\pm 1.2312 \\
\end{array}$ & $\begin{aligned} 19,36 \\
\pm 1.4221 \\
\end{aligned}$ \\
\hline
\end{tabular}

We observed appropriate parallels (i.e. the higher number of clinical uses of bioplastic materials for closure of the nonhealing chronic wounds, the fewer radically mutilating lower limb surgeries) (Figure 2).

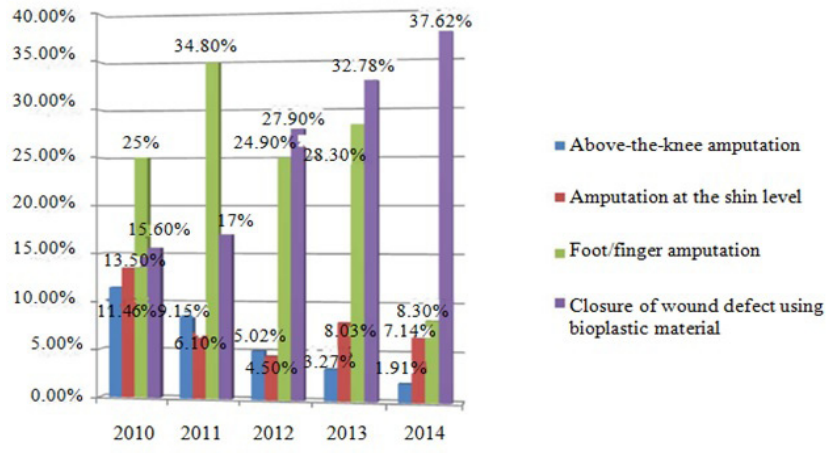

Fig. 2. Dynamics of balance between lower limb amputations and organ preservation surgery at the "Diabetic foot" center in Kazan

\section{Case report 1.}

Patient D., 78-year-old man admitted to the "Diabetic Foot" Center with diagnosis: Type 2 DM, insulin-dependent, severe course, decompensated. DFS, neuroischemic form, W5, diabetic gangrene of the left foot, diabetic angiopathy, CAI stage 4 of the left leg. Photo 1: The 4th day after amputation of the first finger on the left foot with resection at the middle-third of the first metatarsal bone, excision of purulent-necrotic foci of the left foot compartments and necrectomy. The 3 rd day after $\mathrm{X}$-ray endovascular angioplasty of the left lower limb. Photo 2: The 4th day after admission to the "Diabetic Foot" Center. The intra-operative implantation of collagen biomaterial Collost following ultrasonic cavitation with hydrosurgical debridement of the wound surface and exarticulation of the second finger of the left foot. Photo 3: The 35th day after admission to the "Diabetic Foot" Center. Diagnosis: DFS, neuroischemic form, W2, diabetic angiopathy, CAI stage 2a of the left lower limb. Wound epithelialization.

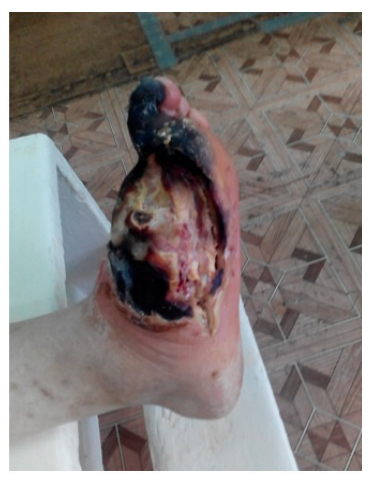

Photo 1.

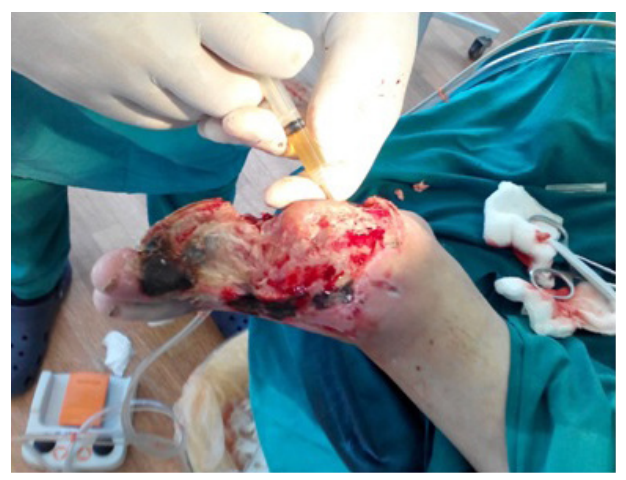

Photo 2.

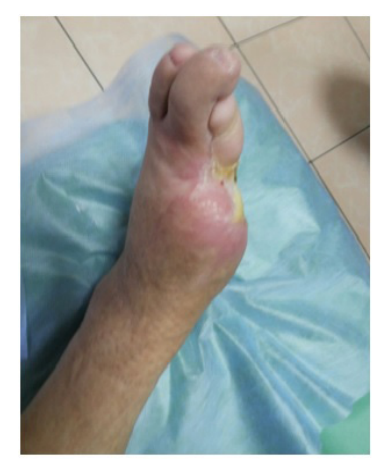

Photo 3. 


\section{Case report 2.}

Patient M., 42-year-old man. Diagnosis: Type 2 diabetes mellitus, insulin-dependent, sub-compensated, severe course. DFS, W2. Trophic ulcers of the right foot. Diabetic angiopathy. CAI stage 3 of the right lower limb. Condition after amputation of the third finger of the right foot with resection of distal head of the third metatarsal bone. Diabetic neuropathy. Diabetic nephropathy. Diabetic retinopathy. Photo 4: View upon admission. Photo 5: Implantation of type I collagen in the form of $7 \%$ gel. Photo 6 : The 27 th day after complex treatment at the "Diabetic Foot" Center and implantation of biomaterial based on type I collagen in the form of membrane and $7 \%$ gel.

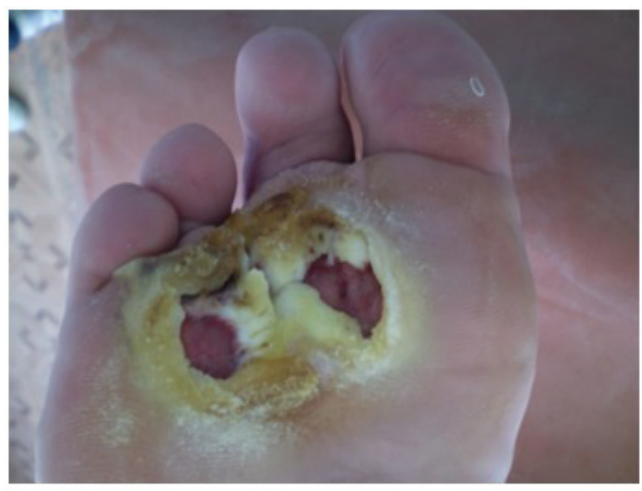

Photo 4.

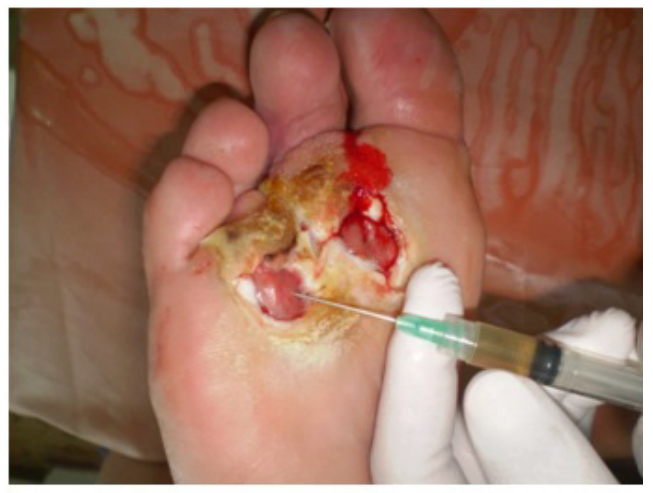

Photo 5.

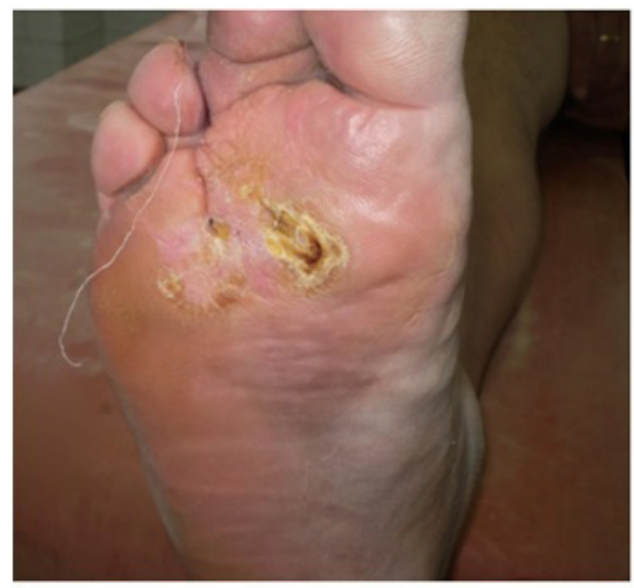

Photo 6.
In conclusion, the use of bioplastic collagen materials in DFS patients enabled us to (1) avoid creating two wound surfaces at the donor and recipient sites for wound defect closure as we do when using autodermoplasty by a free split-thickness skin graft, (2) stimulate tissue regeneration and epithelialization, and (3) reduce the inpatient stay and treatment period. This method can be used for both inpatient and outpatient treatment.

\section{Competing interests}

The authors declare that they have no competing interests.

\section{References}

1. Dedov II, Antsyferov MB, Galstyan GR, Tokmakova AY. Diabetic foot syndrome. Moscow; 1998. [in Russian].

2. Singh N., Armstrong DG, Lipsky BA. Preventing foot ulcers in patients with diabetes. JAMA. 2005; 293(2):217-28. 3. Wagner FW. A classification and treatment program for diabetic neuropathic and dysvascular foot problems. Instr Course Lect 28:143-65, 1979.

4. Report of the Expert Committee on the Diagnosis and Classification of Diabetes Mellitus. Diabetes Care. 1999;22(Suppl 1):S5-S19.

5. Standards of specialized diabetes care. Edited by Dedov II, Shestakova MV. 7th Edition. Diabetes mellitus. 2015;18(1S):1-112. [Article in Russian].

6. Lavery LA, Armstrong DG, Wunderlich RP, Tredwell J, Boulton AJ. Diabetic foot syndrome: evaluating the prevalence and incidence of foot pathology in Mexican Americans and non-Hispanic whites from a diabetes disease management cohort. J Diabetes Care. 2003; 26(5):1435-8.

7. Bregovsky VB, Zaitsev AA, Zapevskaya AG. Lower limb injuries in diabetes mellitus. St. Petersburg: Dilya Publishers, 2004. [in Russian].

8. Chronopoulos A, Tang A, Beglova E, Trackman PC, Roy S. High glucose increases lysyl oxidase expression and activity in retinal endothelial cells: mechanism for compromised extracellular matrix barrier function. Diabetes. 2010;59(12):3159-66.

9. Mazurov VI. Biochemistry of collagen proteins. Moscow, 1974. [in Russian].

10. Ortolan EV, Spadella CT, Caramori C, Machado JL, Gregorio EA, Rabello K.: Microscopic, morphometric and ultrastructural analysis of anastomotic healing in the intestine of normal and diabetic rats. Exp Clin Endocrinol Diabetes 2008;116:198-202 [PubMed]

11. Singh VP, Bali A, Singh N, Jaggi AS. Advanced Glycation End Products and Diabetic Complications. Korean J Physiol Pharmacol. 2014; 18(1): 1-14.

12. El-Mesallamy HO, Hamdy NM, Ezzat OA, Reda AM. Levels of soluble advanced glycation end product-receptors and other soluble serum markers as indicators of diabetic neuropathy in the foot. J Investig Med. 2011;59:1233-1238.

13. Black E, Vibe-Petersen J, Jorgensen LN, Madsen SM, Agren MS, Holstein PE, et al. Decrease of collagen deposition in wound repair in type 1 diabetes independent of glycemic control. Arch Surg. 2003138(1):34-40.

14. Schultz GS1, Wysocki A. Interactions between extracellular matrix and growth factors in wound healing. 
Wound Repair Regen. 2009;17(2):153-62.

15. Briskin BS. Use of bioplastic material Collost in the treatment of wound defects in patients with complicated forms of diabetic foot syndrome. Moscow, 2014. [in Russian].
16. Safoian AA, Nesterenko VG, Nesterenko SV, Alekseeva NU. Bioresorbable collagen matrix, process of its preparation and use. Russian patent 2353397, A61L27/24, A61K35/32, A61K35/36, A61K38/39. 2009. [in Russian]. 